\title{
A Genetic Algorithm for Optimized Reconstruction of Quantized One-dimensional Signals
}

\author{
Frank W. Moore \\ Mathematical Sciences Department \\ University of Alaska Anchorage \\ SSH 154, 3211 Providence Dr., Anchorage, AK 99508 \\ 1-907-786-4819 \\ affwm@uaa.alaska.edu
}

\begin{abstract}
This paper describes a genetic algorithm (GA) that evolves optimized sets of coefficients for one-dimensional signal reconstruction under lossy conditions due to quantization. Beginning with a population of mutated copies of the set of coefficients describing a standard wavelet inverse transform, the genetic algorithm evolves a new set of coefficients that significantly reduces mean squared error (relative to the performance of the selected wavelet) for various classes of onedimensional signals.
\end{abstract}

\section{Categories and Subject Descriptors \\ J.2 [Physical Sciences and Engineering]: Engineering.}

\section{General Terms}

Algorithms, Performance, Experimentation.

\section{Keywords}

Genetic algorithms, wavelets, optimization, evolved transforms.

\section{INTRODUCTION}

Wavelets [5] have been shown to achieve high compression ratios without significant information loss. For many practical problems, however, it is necessary to represent a given signal using a smaller range of possible values. Quantization [2] is the process of mapping signals to a smaller number of bits.

Errors introduced into a transformed signal via quantization may have an unacceptably adverse effect upon the quality of that signal when subsequently reconstructed via the wavelet inverse transform. A growing amount of empirical evidence suggests that non-traditional inverse transformations may do a better job of compensating for the negative effects of quantization, resulting in higher fidelity signal reconstruction. In particular, recent studies suggest that the use of adaptive and/or non-standard filters may significantly reduce quantization error by exploiting characteristics common to specific classes of signals.

The goal of this investigation was to develop a GA capable of modifying the coefficient sets describing a standard wavelet inverse transform to evolve a novel inverse transform exhibiting significantly reduced mean squared error (MSE) for a given class of reconstructed one-dimensional signals. In particular, this GA automatically compensated for errors introduced into the original signal by quantization.

Copyright is held by the author/owner(s).

GECCO'05, June 25-29, 2005, Washington, DC, USA.

ACM 1-59593-010-8/05/0006.

\section{RESULTS}

I conducted a series of tests to demonstrate the performance of GA-evolved transforms relative to that of the Daubechies-4 (Daub4) wavelet inverse transform [1], which is described by the following sets of floating-point coefficients:

$\mathrm{h} 2=\{0.4830,0.8365,0.2241,-0.1294\}$

$\mathrm{g} 2=\{-0.1294,-0.2241,0.8365,-0.4830\}$

Previous research suggested that the solution space in the immediate neighborhood of the Daub4 wavelet was rich with potentially superior combinations of coefficients for novel inverse transforms. For this reason, my GA initialized each of the g2 and h2 coefficients for each inverse transform in generation 0 of each test to a randomly mutated version of the corresponding coefficient from the Daub4 wavelet. Every individual evolved during this investigation thus consisted of two vectors (g2 and $\mathrm{h} 2$ ), each of which contained exactly four floating-point values.

Each test was characterized by a particular combination of the following parameters:

1. SIGNAL CLASS. Signal classes used in this study included ramp functions and sine waves.

2. $\mathbf{G}$ specified the (maximum) number of generations executed by the GA. For each of the tests performed for this study, G $=10000$.

3. M specified the number of candidate solutions in each generation. For each of the tests performed for this study, M $=500$.

4. $\quad \mathbf{P}_{\mathbf{C}}$ specified the percentage of individuals in the next generation subjected to the crossover operator. For each of the tests performed for this study, $\mathrm{P}_{\mathrm{C}}=100 \% *(\mathrm{M}-1) / \mathrm{M}=$ $99.8 \%$.

5. $\quad \mathbf{P}_{\mathbf{M}}$ specified the probability of mutation.

6. $\quad \mathbf{N}$ specified the size of the training population (i.e., the number of signals used to train the GA).

The GA copied the best individual from the current generation into position 0 of the next generation. The GA selected the remaining $\mathrm{M}-1$ individuals from the current generation via tournaments of three randomly-selected individuals. These individuals were then probabilistically subjected to the crossover operator according to $\mathrm{P}_{\mathrm{C}}$. To complete crossover, the GA randomly selected two parent individuals; identified separate crossover points for the g2 and h2 vectors; and exchanged the coefficients from each parent individual located at or beyond each vector's crossover point.

Finally, each coefficient of these M-1 individuals was subjected to a mutation operator with probability $\mathrm{P}_{\mathrm{M}}$, which was allowed to vary dynamically between $0 \%$ and $10 \%$, according to evolutionary progress. The mutation operator multiplied a selected 
coefficient by a small factor F randomly selected from a Gaussian distribution between 0.97 and 1.03; on rare occasion, the mutation operator also negated the coefficient. Restricting the magnitude of the mutation operator in this manner biased the GA to explore the space of non-wavelet transforms immediately adjacent to the Daub4 wavelet.

\subsection{Class 1: Ramp Signals}

Ramp signals are important for a variety of applications [3]. Tests 1,2 , and 3 populated the training set with $\mathrm{N}$ ramp signals, each of which contained 50 sampled values. Table 1 summarizes the performance (as measured by the total MSE for $\mathrm{N}$ reconstructed signals) of each novel inverse transform described by a GAevolved best-of-run coefficient set. These test results demonstrate the following key points:

a) Novel inverse transforms exist that outperform the Daub4 wavelet inverse transform for reconstructing arbitrary ramp signals subjected to quantization error.

b) The GA is capable of automatically optimizing coefficient sets for these novel inverse transforms.

\subsection{Class 2: Sine Waves}

The detection of periodic behavior in one-dimensional signals continues to be a research topic of considerable importance [4]. Tests 4 through 12 populated the training set with various types of sine waves characterized by three parameters: frequency (f), gain $(\mathbf{g})$, and offset $(\mathbf{d})$. For these tests, each vector $\mathbf{v}$ consisted of 50 sampled values. Table 2 summarizes the performance of GAevolved best-of-run coefficient sets optimized under conditions described by various combinations of $\mathbf{f}, \mathbf{g}$, and $\mathbf{d}$, relative to that of the Daub4 wavelet described above. Tests 4, 5, and 6 demonstrated that the GA was capable of identifying coefficients for inverse transforms that significantly outperformed the Daub4 inverse transform for the task of reconstructing sine waves characterized by different $\mathbf{f}$ values. For this signal class, GAoptimized transforms were capable of reducing MSE in the reconstructed signal by up to $97.2 \%$.

Tests 7,8, and 9 showed that, when the training set consisted of sine waves that differed only according to the offset $\mathbf{d}$, little advantage was to be gained from evolving novel coefficients for inverse transforms. For these three tests, the performance of the inverse transforms described by the evolved coefficient sets improved upon that of the Daub4 inverse transform by an average of only $2.17 \%$.

Tests 10,11, and 12 demonstrated the GA's ability to evolve coefficients for inverse transforms that significantly outperformed the Daub4 inverse transform for the task of reconstructing sine waves that differed only in the gain value g. For this class of signal, GA-optimized inverse transforms were capable of reducing MSE in the reconstructed signal by $90.6 \%$ or more.

\section{CONCLUSIONS}

Collectively, the results of this study suggested that the number of coefficient sets capable of producing high-fidelity signal reconstructions under lossy conditions may be much larger than previously believed. The novel coefficient sets evolved during this study violated wavelet properties required for perfect reconstruction, such as invertibility and non-redundancy. Nevertheless, the corresponding inverse transforms consistently outperformed the Daub4 inverse transform, producing significantly higher fidelity reconstructions of periodic signals, as measured by the percentage reduction in the MSE of each reconstructed signal. The results of this study strongly encouraged the identification and use of evolved inverse transforms for signal reconstruction under lossy conditions subject to quantization.

\section{REFERENCES}

[1] Daubechies, I. 1992. Ten Lectures on Wavelets, SIAM.

[2] Mallat, S. 1998. A Wavelet Tour of Signal Processing, Academic Press.

[3] Nag, S. K. and L. Peters, Jr. 1998. Ramp Response Signatures for Dielectric Targets, SPIE Proceedings, 3392: 703-713, SPIE.

[4] Venkatachalam, V. and J. Aravena 1998. Detecting Periodic Behavior in Nonstationary Signals, IEEE-SP International Symposium on Time-frequency and Time-scale Analysis, IEEE.

[5] Walker, J. S. 1999. A Primer on Wavelets and Their Scientific Applications, CRC Press.

Table 1. Ramp Signal Test Results

\begin{tabular}{|c|c|c|c|c|}
\hline$\underline{\text { Test }}$ & $\underline{N}$ & MSE (Daub4) & MSE (evolved) & \% Improvement \\
\hline 1 & 100 & 5505.1 & 4838.9 & $12.1 \%$ \\
\hline 2 & 25 & 1376.0 & 1215.0 & $11.7 \%$ \\
\hline 3 & 10 & 552.4 & 522.9 & $5.35 \%$ \\
\hline
\end{tabular}

Table 2. Sine Wave Test Results

\begin{tabular}{|c|c|c|c|c|c|c|c|}
\hline Test & $\underline{\mathrm{N}}$ & g & $\underline{\mathbf{d}}$ & $\underline{\mathbf{f}}$ & MSE (Daub4) & MSE (evolved) & $\%$ Improvement \\
\hline$\overline{4}$ & $1 \overline{0} 0$ & 128 & $2 \overline{5} 6$ & $0 . . \overline{999}$ & 1096.19 & 86.69 & $92.1 \%$ \\
\hline 5 & 25 & 128 & 256 & $0 \ldots 999$ & 269.00 & 20.18 & $92.5 \%$ \\
\hline 6 & 10 & 128 & 256 & $0 \ldots 999$ & 124.55 & 3.55 & $97.2 \%$ \\
\hline 7 & 100 & 128 & $0 \ldots 255$ & 1000 & 5207.62 & 5034.13 & $3.33 \%$ \\
\hline 8 & 25 & 128 & $0 \ldots 255$ & 1000 & 1203.68 & 1182.69 & $1.74 \%$ \\
\hline 9 & 10 & 128 & $0 \ldots 255$ & 1000 & 403.67 & 397.82 & $1.45 \%$ \\
\hline 10 & 100 & $0 \ldots 127$ & 256 & 1000 & 1123.89 & 88.19 & $92.2 \%$ \\
\hline 11 & 25 & $0 \ldots 127$ & 256 & 1000 & 274.49 & 24.90 & $90.9 \%$ \\
\hline 12 & 10 & $0 \ldots 127$ & 256 & 1000 & 108.94 & 10.30 & $90.6 \%$ \\
\hline
\end{tabular}

